\title{
Integer quantum Hall effect in single-layer graphene with tilted magnetic field
}

\author{
P. M. Krstajić \\ Institute of Microelectronic Technologies and Single Crystals (IHTM), University of Belgrade, Njegoševa 12, \\ 11000 Belgrade, Serbia
}

(Received 7 May 2013; accepted 1 August 2013; published online 16 August 2013)

\begin{abstract}
Analytical expression for the Hall conductivity $\sigma_{y x}$ in graphene is derived in more general case, in which magnetic field is not perpendicular to the plane of the sample. The derivations were carried out using Kubo-Greenwood formulism, which is more suitable when the spectrum is discrete. The results are analyzed as a function of the angle between the magnetic field vector and the normal of the graphene plane. Contrary to the previous theoretical studies, we show that the plateau levels are independent of the direction of the magnetic field. The positions of the plateaus, however, depend on the orientation of the magnetic field. (C) 2013 AIP Publishing LLC. [http://dx.doi.org/10.1063/1.4818605]
\end{abstract}

\section{INTRODUCTION}

The discovery of graphene ${ }^{1}$ is certainly one of the most important events in condensed matter physics in recent time. The reason in increased interest in this material lies in its remarkable properties ${ }^{2}$ such as high (room temperature) electron mobility, ${ }^{3}$ good thermal conductivity, ${ }^{4}$ and exceptional mechanical properties. ${ }^{5}$ One of the most striking phenomena observed is the anomalous and integer quantum Hall effect (QHE) which is astonishingly attainable phase in this material even at room temperature. ${ }^{6}$ The latter is possible due to the large inter Landau level spacings compared to the thermal energy $k_{B} T$. However, QHE in graphene has somewhat different manifestation than in conventional semiconductors, one of the most important being the absence ${ }^{7,8}$ of a Hall plateau when the Fermi level crosses the Dirac point. This is due to the fact that the zeroth level is equally shared by the conduction and the valence band.

In this paper, we investigate the more general case of experimental setup for QHE, in which the magnetic field has an arbitrary orientation with respect to the sample plane. It was shown that the expression for the Hall conductivity is analogous to that in the usual case, but of course depends on the angle $\theta$ between the field and the plane normal. This somewhat contradicts the previous theoretical investigation, ${ }^{9}$ where the derivations were done under certain approximations.

The paper is organized as follows. The theoretical model for quantum Hall state in a graphene with arbitrary magnetic field orientation is presented in Sec. II. Subsequently, a detailed derivation of the expression for the Hall conductivity $\sigma_{y x}$ is given. Numerical results for $\sigma_{y x}$ and longitudinal resistivity $\rho_{x x}$ as a function of parameters are presented and discussed in Sec. III. Finally, Sec. IV summarizes the main results.

\section{THEORETICAL MODEL}

Let us assume that the graphene sample lies in the $x-y$ plane while the magnetic field forms an angle $\theta$ with the normal to the plane, thus, with the $z$-axis. The magnetic field will inevitably induce a gap in the energy spectrum, and one should treat the two valleys, $K$ and $K^{\prime}$ separately. The Hamiltonian written in $(2+1)$ dimensions reads

$$
H_{\xi}=v_{F}\left(\xi \sigma_{x} \pi_{x}+\sigma_{y} \pi_{y}\right)+\Delta_{z} \sigma_{z},
$$

where $v_{F}$ is the Fermi velocity, $\sigma_{\alpha}$ with $\alpha=x, y, z$ are the Pauli matrices, while $\xi$ is the valley pseudospin number with $\xi=+1$ for the $K$ valley and $\xi=-1$ for the $K^{\prime}$ valley. Further $\pi_{x, y}$ are the momenta in the presence of a magnetic field with $\pi_{x, y}=p_{x, y}+e A_{x, y}$. The Landau gauge is chosen and $\mathbf{A}=(-B \cos \theta \cdot y, 0)$. The second term in Eq. (1) represents the pseudo-Zeeman term, and may also include the mass term $\Delta_{0}$ induced by the substrate $\Delta_{z}=-\mu_{B} B \cos \theta+\Delta_{0}$, where $\mu_{B}$ is the Bohr magneton. The normalized eigenvectors of the eigenvalue problem $H_{\xi} \Psi=E \Psi$ are (see the Appendix)

$$
\Psi_{s n k_{x}}=\frac{1}{\sqrt{L_{x}}}\left(\begin{array}{c}
a_{s n} \Phi_{n-1} \\
s b_{s n} \Phi_{n}
\end{array}\right) e^{i k_{x} x}
$$

where $\Phi_{n}\left(y_{0}\right)$ are the quantum oscillator functions with $y_{0}=\left(y-l_{c}^{2} k_{x}\right) / l_{c}$. Here, the magnetic length is $l_{c}=\sqrt{\hbar /(B \cos \theta)}$. The index $\xi$ will be suppressed form now on. The quantum number $n(n=0,1,2, .$.$) labels the discrete$ Landau levels while $s$ labels the conduction $(s=+1)$ and the valence bands $(s=-1)$. The latter is related to the valley pseudospin quantum number by $s=\xi \eta$ where $\eta$ is the chirality. The eigenvalues of Eq. (1) are expressed in terms of the cyclotron frequency which in its turn (in case of graphene) is proportional to the square root value of the magnetic field $\omega_{c}=\sqrt{2} v_{F} / l_{c} \propto \sqrt{B \cos \theta}$. They are given by

$$
E_{s n}=s\left[\Delta_{z}^{2}+n \hbar^{2} \omega_{c}^{2}\right]^{1 / 2}\left(1-\delta_{n 0}\right), E_{s 0}=-\xi \Delta_{z} \delta_{n 0} .
$$

In the presence of a gap $\Delta_{z}$, the coefficients $a_{s n}$ and $b_{s n}$ are related to the energy $E_{s n}$ in the following manner:

$$
a_{s n}=\sqrt{\frac{E_{s n}+\Delta_{z}}{2 E_{s n}}}, \quad b_{s n}=\sqrt{\frac{E_{s n}-\Delta_{z}}{2 E_{s n}}} .
$$


The case of the zeroth level $\left|s, 0, k_{x}\right\rangle$ requires special care and will be considered separately. The value of the usual Zeeman term $g \mu_{B} B \cos \theta$ is (at most) on the order ${ }^{2} 10 \mathrm{~K}$ for $B \approx 10 \mathrm{~T}$, and thus, negligible. Further, if one neglects the influence of substrate the zero-energy level is placed at the Dirac point and is then equally shared between the conduction and valence bands. However, in the case of interest, one should consider both valleys separately. The normalized eigenvector that corresponds to this state has the form:

$$
\Psi_{s 0 k_{x}}=\frac{1}{\sqrt{L_{x}}}\left(\begin{array}{c}
0 \\
\Phi_{0}
\end{array}\right) e^{i k_{x} x}
$$

Notice that $\left|+, 0, k_{x}\right\rangle$ and $\left|-, 0, k_{x}\right\rangle$ denote the same state. The sign of $E_{s 0}$ that corresponds the zeroth level energy is negative for the $K$ valley and positive for the $K^{\prime}$ valley.

The electric, source-drain field is usually weak and one may employ linear response theory ${ }^{10}$ and obtain the Hall conductivity in the form: ${ }^{11}$

$$
\sigma_{\mu \nu}^{n d}=\frac{i \hbar e^{2}}{S_{0}} \sum_{\zeta \neq \zeta^{\prime}} \frac{\left(f_{\zeta}-f_{\zeta^{\prime}}\right) v_{\nu \zeta^{\prime} \zeta^{\prime}} v_{\mu \zeta^{\prime \prime} \zeta}}{\left(E_{\zeta}-E_{\zeta^{\prime}}\right)\left(E_{\zeta}-E_{\zeta^{\prime}}+i \Gamma_{\zeta}\right)},
$$

where $S_{0}$ is the area of the sample, and $v_{\nu, \mu \zeta \zeta^{\prime}}$ are the matrix elements of the velocity operator with $\mu, \nu=x, y$. Further $f_{\zeta}=f\left(E_{\zeta}\right)$ is the Fermi-Dirac distribution function, $f\left(E_{\zeta}\right)=\left(1+\exp \beta\left(E-E_{F}\right)\right)^{-1}$, while the symbol $\beta$ denotes the Boltzmann factor $\beta=1 / k_{B} T$ ( $T$ is the temperature). The sum runs over all quantum numbers $|\zeta\rangle=\left|s, n, k_{x}\right\rangle$ and $\left|\zeta^{\prime}\right\rangle=\left|s^{\prime}, n^{\prime}, k_{x}^{\prime}\right\rangle$ provided that $\zeta \neq \zeta^{\prime}$. The infinitesimal quantity $\epsilon$ in the usual form ${ }^{10}$ has been replaced by $\Gamma_{\zeta}$ in order to take into account the finite broadening of the Landau levels. The broadening of the levels is mainly caused by the collisions on impurities. It can be proven that the imaginary part of Eq. (6) vanishes under assumption that the broadening is approximately the same for all states, ${ }^{12} \Gamma_{\zeta}=\Gamma$. Further, it was shown ${ }^{12,13}$ that $\Gamma$ increases with the magnetic field like $\propto \sqrt{B}$, or more precisely ${ }^{12} \Gamma=\sqrt{2 / A} \hbar \omega_{c}$. Here, the dimensionless and phenomenological parameter $A$ depends on the concentration of scattering centers and has values in the range $50-100$. In order to obtain a transparent result for $\sigma_{y x}$, we assume that $\Gamma=0$.

One needs to evaluate the product of the velocity matrix elements which are diagonal in $k_{x}$

$$
P_{n n^{\prime}}^{s \prime^{\prime}}=\left\langle s n k_{x}\left|v_{x}\right| s^{\prime} n^{\prime} k_{x}^{\prime}\right\rangle\left\langle s^{\prime} n^{\prime} k_{x}^{\prime}\left|v_{y}\right| s n k_{x}\right\rangle
$$

The quantum number $k_{x}$ is suppressed from now on. An explicit evaluation of the product $P_{n n^{\prime}}^{s s^{\prime}}$ yields

$$
P_{n n^{\prime}}^{s \prime^{\prime}}=i v_{F}^{2}\left(\left|a_{s n} b_{s^{\prime} n^{\prime}}\right|^{2} \delta_{n-1, n^{\prime}}-\left|a_{s^{\prime} n^{\prime}} b_{s n}\right|^{2} \delta_{n, n^{\prime}-1}\right) .
$$

The matrix elements between the zeroth level and the other levels should be treated separately. ${ }^{11}$ Using Eqs. (7) and (8), one arrives at

$$
P_{0 n^{\prime}}^{s s^{\prime}}=-i v_{F}^{2}\left|a_{s^{\prime} n^{\prime}}\right|^{2} \delta_{0, n^{\prime}-1}, \quad P_{n 0}^{s s^{\prime}}=i v_{F}^{2}\left|a_{s n}\right|^{2} \delta_{n-1,0} .
$$

Since the relation $\left|E_{s n}\right|>\Delta_{z}$ holds for $n>0$ and moderate magnetic fields, one may omit the complex modulus signs in Eq. (9). First, we assume that $\Gamma_{\zeta}=\Gamma=0$. The summation in Eq. (6) should include all combinations of the matrix elements, which is to encompass both the conduction/ valence band and the two valleys. The Hall conductivity $\sigma_{\xi}$ for a particular valley can be expressed as a sum of two terms, in one of which the prefactor is independent of $\Delta_{z}$ and a term in which it is linear in $\Delta_{z}, \sigma_{\xi}=\sigma_{\xi 0}+\delta \sigma_{\xi}$. It can be proven ${ }^{14}$ that for the valley $K$ and for $n>0$, the relevant expressions are

$$
\sigma_{K 0}=\frac{g_{s} e^{2}}{h} \sum_{n=1}\left(n+\frac{1}{2}\right)\left(f_{n}^{+}-f_{n+1}^{+}+f_{n}^{-}-f_{n+1}^{-}\right),
$$

while the term proportional to the mass term $\Delta_{z}$ is

$$
\delta \sigma_{K}=\frac{g_{s} e^{2}}{h} \Delta_{z} \sum_{n=1}\left(\frac{f_{n}^{+}-f_{n}^{-}}{E_{n}}-\frac{f_{n+1}^{+}-f_{n+1}^{-}}{E_{n+1}}\right) .
$$

The factor $g_{s}$ is the spin degeneracy, $g_{s}=2$. One should remember that these derivations are given for carriers in the vicinity of the $K$ point only. In order to include the carriers near the $K^{\prime}$ point as well, one should replace $\Delta_{z}$ by $-\Delta_{z}$ in Eq. (11). Since the factor $\Delta_{z}$ appears in the first and odd power in Eq. (11), the terms $\delta \sigma_{K}$ will cancel for $n>0$. For $n=0$, it can be proven that the contribution from the zeroth level is $\sigma_{y x}^{0, \xi}=g_{s} e^{2} / h f_{0}^{\xi}=g_{s} e^{2} /(2 h)\left(f_{0}^{+, \xi}+f_{0}^{-, \xi}\right)$. Bear in mind that $f_{0}^{+, \xi}=f_{0}^{-, \xi}=f_{0}^{\xi}$ for each valley $\xi$, while the superscript \pm in $f_{0}^{ \pm, \xi}$ is not to be confused with the actual sign of the zeroth level in a particular valley. Therefore, this level can be included in the final expression for $\sigma_{y x}$, without worrying that some terms will be summed twice. Finally,

$$
\sigma_{y x}^{n d}=\frac{g_{s} e^{2}}{h} \sum_{n=0, \xi}\left(n+\frac{1}{2}\right)\left(f_{n}^{+, \xi}-f_{n+1}^{+, \xi}+f_{n}^{-, \xi}-f_{n+1}^{-, \xi}\right) .
$$

The summation over $\xi$ has been performed as the zeroth levels of the $K$ and $K^{\prime}$ valleys do not coincide. Note that the form of Eq. (12) is the same as in the case of a perpendicular magnetic field. The degeneracy with respect to the spin degree of freedom is, as usual $g_{s}=2$. Note that Eq. (12) turns into expression derived by Gusynin and Sharapov ${ }^{8}$ by virtue of the identity $\tanh \left(\beta\left(E-E_{F}\right) / 2\right)=1-2 f(E)$. Furthermore, at low temperatures and at moderate magnetic fields, Eq. (12) assumes a simple form ${ }^{7,11} \sigma_{y x}= \pm\left(4 e^{2} / h\right)$ $(N+1 / 2)$, where $N$ is a non-negative integer and is in fact the number of filled levels. This expression for the Hall conductivity can be written in terms of the filling factor $\nu$, $\sigma_{y x}=\nu e^{2} / h$, and therefore, ${ }^{15} \nu=4 N+2$. The presence of the factor $1 / 2$ is an obvious manifestation of the anomalous IQHE in (gapless) samples of graphene. This phenomenon can be explained by the existence of a Landau level at the Dirac point equally shared by the conduction and valence bands. However, since early days of research of quantum Hall phenomena in semiconductors, a particular attention is given to disorder and imperfections. It is known that the existence of plateaus cannot be explained without the assumption that there are impurities and disorder present in the 
material. ${ }^{16}$ A detailed study made by Ostrovsky ${ }^{17}$ emphasized the role of the symmetry of the disorder in the preservation of the quantum Hall state in graphene.

Next we consider the longitudinal conductivity $\sigma_{x x}$, which is another parameter that characterizes magnetotransport properties of materials. In the presence of a magnetic field, the diffusive contribution to the conductivity vanishes $\sigma_{x x}^{d i f}=0$ and only the collisional contribution $\sigma_{x x}^{c o l}$ is relevant. The latter is given by ${ }^{10}$

$$
\sigma_{x x}^{c o l}=\frac{\beta e^{2}}{2 S_{0}} \sum_{\zeta, \zeta^{\prime}} f\left(E_{\zeta}\right)\left[1-f\left(E_{\zeta^{\prime}}\right)\right] W_{\zeta \zeta^{\prime}}\left(y_{\zeta}-y_{\zeta^{\prime}}\right)^{2},
$$

where $W_{\zeta \zeta^{\prime}}$ is the transition rate between the states $|\zeta\rangle$ and $\left|\zeta^{\prime}\right\rangle$, while $y_{\zeta}=\langle\zeta|y| \zeta\rangle$ is the expectation value of the coordinate $y$. It is assumed that scattering by impurities is assumed to be elastic, while the impurity potential is screened $U(\mathbf{r})=U_{0} e^{-k_{s} r}$, where $k_{s}$ is the screening wavevector. The concentration of impurities is $n_{i}$. It can be proven ${ }^{14}$ that longitudinal conductivity is

$$
\sigma_{x x}^{c o l}=\frac{g_{s} e^{2}}{h} \frac{\beta n_{i} U_{0}^{2}}{4 u_{c s} \hbar \omega_{c}} \sum_{s, n, \xi} I_{s n} f\left(E_{s n}^{\xi}\right)\left[1-f\left(E_{s n}^{\xi}\right)\right]
$$

where $u_{c s}=l_{c}^{2} k_{s}^{2} / 2$, and

$$
I_{s n}=(2 n+1)\left|b_{s n}\right|^{4}-2 n\left|a_{s n}\right|^{2}\left|b_{s n}\right|^{2}+(2 n-1)\left|a_{s n}\right|^{4} .
$$

In case when $\Delta_{z}=0$, the expression for $I_{s n}$ reduces to $2 n / 4$, and it means that the minima of $\sigma_{x x}$ occur at odd filling factors $\nu=2 n+1$. Bear in mind that in common semiconductors the minima occur at even filling factors $\nu=2 n$, and that $\sigma_{x x} \propto(2 n+1)$.

\section{NUMERICAL RESULTS}

First, the Hall conductivity $\sigma_{y x}$ vs the magnetic field is shown in Fig. 1 for fixed electron concentration $n_{e}$ $=10^{12} \mathrm{~cm}^{-2}$, for two different values of the angle $\theta$. The solid curve corresponds to the usual case when the magnetic field is perpendicular to the sample plane, $\theta=0^{\circ}$ while the dashed to the case in which $\theta=45^{\circ}$. The difference between the two curves is mostly pronounced in the positions of the plateaus, i.e., in the values of magnetic field when a new plateau emerges. This is consequence of the presence of the factor $\cos \theta$ in the cyclotron radius $l_{c}$, cyclotron energy, etc. The influence through the mass term $\Delta_{z}$ is less marked since its value is on the order of several $\mathrm{meV}$. As it can be seen from the figure for the larger angle $\theta=45^{\circ}$, the plateaus are shifted to the right, since higher magnetic fields are needed to compensate the factor $\cos \theta$. On the other hand, the heights of the plateaus are integer multiplies of $e^{2} / h$ regardless of the angle $\theta$.

In the next figure, Fig. 2 is shown the conductivity $\sigma_{y x}$ as a function of the electron concentration $n_{e}$, but for various values of the angle $\theta: 0^{\circ}$ (solid line), $30^{\circ}$ (dotted line), $45^{\circ}$ (dashed line), and $60^{\circ}$ (dashed-dotted line). The magnetic field is kept constant at $B=14 \mathrm{~T}$. As can be seen from the figure, the plateaus again come in multiple integers of $e^{2} / h$,

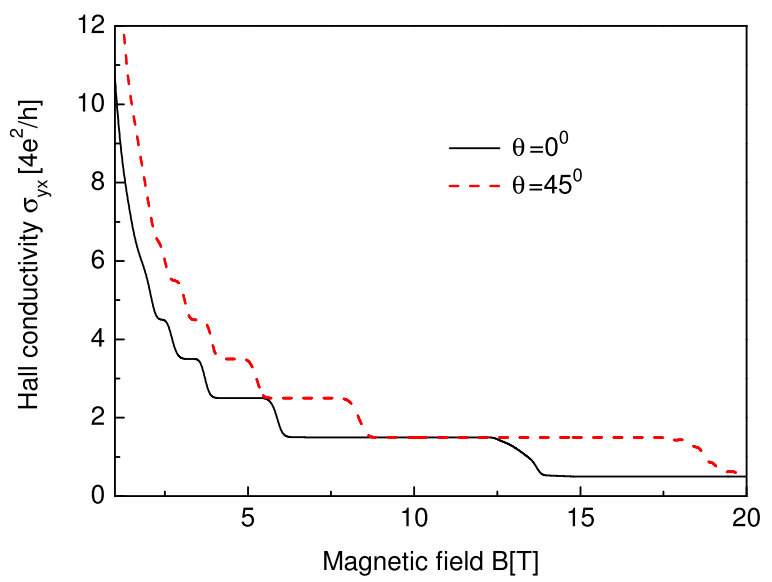

FIG. 1 . The Hall conductivity $\sigma_{y x}$ vs the magnetic field at fixed electron concentration $n_{e}=10^{12} \mathrm{~cm}^{-2}$, but for two different values of the angle $\theta: 0^{\circ}$ (solid black curve) and $45^{\circ}$ (dashed red curve).

but their onset (as $n_{e}$ rises) depends on the angle $\theta$. This can be explained by the fact that the areal density of states for each level is $N_{L L}=g_{d} e B \cos \theta / h$ while there are $n_{e}$ electrons (per unit area) available to fill these levels. Here, $g_{d}$ is the degeneracy, which for $n=0$ and $n>0$ takes on values 2 and 4 , respectively. Therefore, with the increase $\theta$, the density $N_{L L}$ decreases, and consequently, the number of filled levels rises. That is why the curves with higher $\theta$ look as if moved to the origin with respect to the standard case $\theta=0$. Note the absence of a plateaux at $n_{e}=0$ in contrast what was found in Refs. 11 and 14. The reason is that the main subject of this paper is to investigate the influence of the tilted magnetic field on QHE state and thus the scattering on impurities is neglected. Therefore, there is no shift in the energy spectrum at the Dirac point.

Next figure, Fig. 3 shows the conductivity $\sigma_{y x}$ as a function of the Fermi energy $E_{F}$, for three different values of the angle $\theta$ like in the previous case. The magnetic field is kept constant at the value $B=14 \mathrm{~T}$. This kind of graph is important when the value of the Fermi energy is known with a good accuracy, or when the dependence $E_{F}\left(n_{e}\right)$ is not known precisely. For illustrative purposes, we also give the case

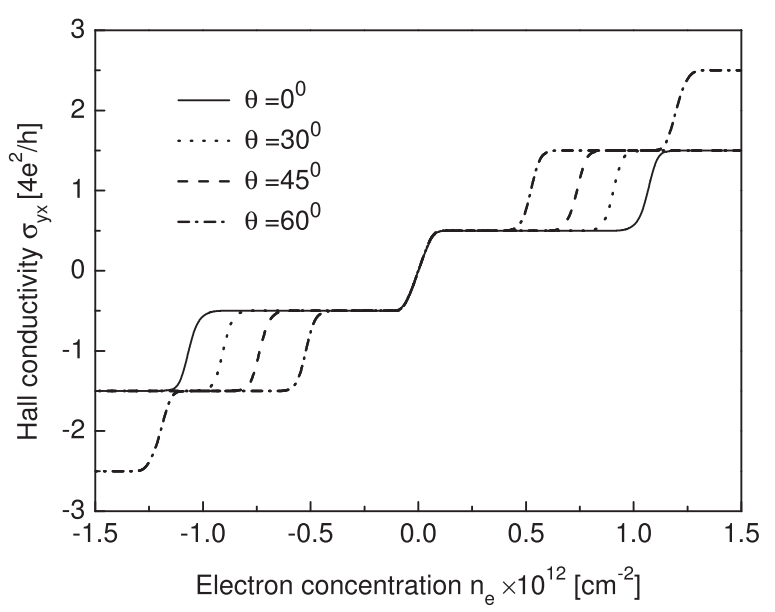

FIG. 2. The Hall conductivity $\sigma_{y x}$ vs the electron concentration $n_{e}$, at fixed magnetic field $B=14 \mathrm{~T}$, but for various values of $\theta: 0^{\circ}$ (solid line), $30^{\circ}$ (dotted line), $45^{\circ}$ (dashed line), and $60^{\circ}$ (dashed-dotted line). 


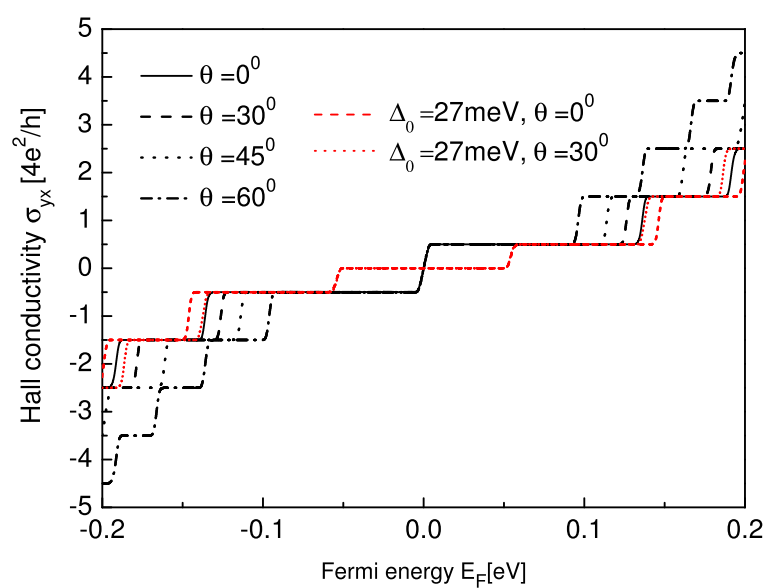

FIG. 3. The Hall conductivity $\sigma_{x y}$ vs the Fermi energy $E_{F}$, for three different values of $\theta: 0^{\circ}$ (solid curve), $30^{\circ}$ (dashed curve), $45^{\circ}$ (dotted curve), and $60^{\circ}$ (dashed-dotted curve). For comparison, another arrangement when the substrate induces an additional gap, $\left(\Delta_{0}=27 \mathrm{meV}\right)$ is given by the red (short) dashed $\left(\theta=0^{\circ}\right)$ and dotted curve $\left(\theta=30^{\circ}\right)$. In all cases, the magnetic field is kept constant at the value $B=14 \mathrm{~T}$.

when the substrate induces additional gap between the conduction and the valence band, that is when $\Delta_{0}>0$. Namely, in recent time, there is a growing interest in graphene placed on hexagonal boron nitride $(\mathrm{h}-\mathrm{BN})^{18-22}$ and silicon carbide $(\mathrm{SiC})^{23,24}$ both of which induces an appreciable gap. The gap can be important since the device based on this kind of graphene samples may have rectifying properties as much as if they were made of a conventional semiconductor. As it can be seen from the figure, the gap induces a plateau at the $E_{F}=0$, a phenomenon that is also suggested by available experimental data. ${ }^{18}$ In the present graph, we have chosen hBN substrate with a moderate value ${ }^{21}$ of $2 \Delta_{0}=53 \mathrm{meV}$. It can be deduced from the figure that cases with $\Delta_{0} \neq 0$ follow the usual case when $\Delta_{0}=0$, but the curves are bit moved due to different energy level positions.

As far as longitudinal transport quantities are concerned, we note that longitudinal resistivity $\rho_{x x}$ is experimentally measurable quantity and is given by

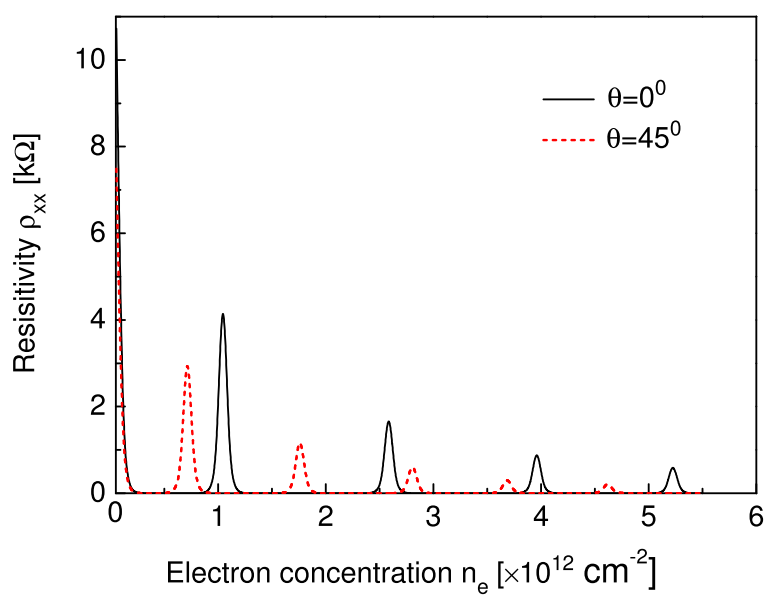

FIG. 4. The longitudinal resistivity $\rho_{x x}$ vs the electron concentration $n_{e}$, for two values of $\theta: 0^{\circ}$ (solid curve) and $45^{\circ}$ (short-dashed curve). The magnetic field strength is kept constant at the value $B=14 \mathrm{~T}$.

$$
\rho_{x x}=\frac{\sigma_{x x}}{\sigma_{x x}^{2}+\sigma_{y x}^{2}} .
$$

Due to the presence of the Hall conductivity in the previous relation, Eq. (16), the value of $\rho_{x x}$ is influenced by the plateau values in $\sigma_{y x}$. Figure 4 shows the resistivity $\rho_{x x}$ as a function of the electron concentration $n_{e}$ for two values of the angle $\theta: 0^{\circ}$ (solid curved) and $45^{\circ}$ (short dashed curve). The value of the magnetic field is kept constant $B=14 \mathrm{~T}$. The oscillatory behavior is apparent as a function of the electron concentration. The position of peaks coincide closely to transitional phases or onsets of plateaus in $\sigma_{y x}$. The values of $\rho_{x x}$ are higher when perpendicular magnetic field is applied $\left(\theta=0^{\circ}\right)$ while peaks are moved to the left for finite angle $\theta$. The latter fact can also be deduced from Fig. 2 where plateaus arise for smaller $n_{e}$.

\section{CONCLUDING REMARKS}

In this work, we investigated the Hall conductivity in graphene when the magnetic field is not perpendicular to the plane of graphene. It was shown that its value comes in multiple integers of $e^{2} / h$ like in the usual case, but the onset of the plateaus appears for higher magnetic field and lower electron concentrations if the angle between $\mathbf{B}$ and the plane normal is increased. This is due to the fact that the (available) density of states of each state is $g_{d} e B \cos \theta / h$ and thus decreases with the increase of the angle $\theta$. This was also shown analytically by summing the contribution of both valleys carefully, when the influence of the gap is canceled. We also give the case when there is an additional source of gap (apart from the magnetic field). Namely, when a graphene is placed on a substrate like $\mathrm{h}-\mathrm{BN}$ or $\mathrm{SiC}$ a staggered potential is formed in the crystal structure. This means that the on-site energies of the carbon atoms belonging to the A and B sublattices have different values, denoted by $+\Delta_{0}$ and $-\Delta_{0}$, respectively. The calculations given here indicate that this kind of substrate induces an additional plateau at $E_{F}=0$, while other properties of $\sigma_{y x}$ remain qualitatively the same as in case when $\Delta_{0}=0$. At the end, we evaluate and plot the longitudinal resistivity as a function of the electron concentration. It was shown that the positions and the values of maxima of $\rho_{x x}$ depend on the value of the angle between the magnetic field and the normal of the graphene plane.

\section{ACKNOWLEDGMENTS}

This work was supported by the Serbian Ministry of Education and Science, within the Project No. TR 32008.

\section{APPENDIX: EIGENVECTORS IN TILTED MAGNETIC FIELD}

The Hamiltonian, Eq. (1) can be expressed in terms of the annihilation and creation operators, and for the $K$ valley, it reads

$$
H=\left(\begin{array}{cc}
\Delta_{z} & \hbar \omega_{c} a \\
\hbar \omega_{c} a^{\dagger} & -\Delta_{z}
\end{array}\right)
$$


where $a=l_{c} /(\sqrt{2} h)\left(\pi_{x}-i \pi_{y}\right)$ and $a^{\dagger}=l_{c} /(\sqrt{2} h)\left(\pi_{x}+i \pi_{y}\right)$. Here, momenta $\pi_{\mu}$ are defined as $\pi_{\mu}=p_{\mu}+e A_{\mu}$ with $\mu=x, y$. If the general form of the eigenvector $\Psi=\left(u_{n}, v_{n}\right)^{T}$ is inserted in the equation $H \Psi=E \Psi$, one obtains

$$
a^{\dagger} a v_{n}=\frac{E_{s n}^{2}-\Delta_{z}^{2}}{\left(\hbar \omega_{c}\right)^{2}} \cdot v_{n} .
$$

Thus, $v_{n}$ is the eigenfunction of the number operator $a^{\dagger} a$, while $u_{n} \propto a v_{n}$. This suggests the following form for eigenvectors when $n>0$ :

$$
\Psi_{s n k_{x}}=\frac{1}{\sqrt{L_{x}}}\left(\begin{array}{c}
a_{s n} \Phi_{n-1} \\
s b_{s n} \Phi_{n}
\end{array}\right) e^{i k_{x} x} .
$$

The eigenvalue problem leads to the system of two equations for the coefficients $a_{s n}$ and $b_{s n}$

$$
\begin{aligned}
& a_{s n} \Delta_{z}+s \sqrt{n} \hbar \omega_{c} b_{s n}=E_{s n} a_{s n} \\
& a_{s n} \sqrt{n} \hbar \omega_{c}-s \Delta_{z} b_{s n}=s E_{s n} b_{s n} .
\end{aligned}
$$

Now one can determine the coefficients in the eigenvector given the normalization condition $\left|a_{s n}\right|^{2}+\left|b_{s n}\right|^{2}=1$. The expressions read

$$
a_{s n}=\sqrt{\frac{E_{s n}+\Delta_{z}}{2 E_{s n}}}, \quad b_{s n}=\sqrt{\frac{E_{s n}-\Delta_{z}}{2 E_{s n}}} .
$$

The eigenenergies can be obtained by eliminating $a_{s n}$ and $b_{s n}$ in Eqs. (A4a) and (A4b) and read $E_{s n}=s \sqrt{\Delta_{z}^{2}+n \hbar^{2} \omega_{c}^{2}}$. The case of the zeroth level should be treated separately. One may infer from Eq. (A3) that the first component should be zero and

$$
\Psi_{s 0 k_{x}}=\left(\begin{array}{c}
0 \\
\Phi_{0}
\end{array}\right) e^{i k_{x} x},
$$

while the corresponding energy is $E_{0}=-\Delta_{z}$. The eigenvectors for the another valley $K^{\prime}$ can be obtained by the interchange of the spinor components $u_{n} \leftrightarrow v_{n}$ which is the consequence of the chiral symmetry of charge carriers in graphene. The eigenvectors, Eq. (A3) can be brought to the form given in Ref. 9 by using the trigonometric identities of half angle (up to the overall phase factor).

${ }^{1}$ K. S. Novoselov and A. K. Geim, Science 306, 666 (2004).

${ }^{2}$ A. H. Castro Neto, F. Guinea, N. M. R. Peres, K. S. Novoselov, and A. K. Geim, Rev. Mod. Phys. 81, 109 (2009).

${ }^{3}$ K. I. Bolotin, K. J. Sikes, Z. Jiang, M. Klima, G. Fudenberg, J. Hone, P. Kim, and H. L. Stormer, Solid State Commun. 146, 351 (2008).

${ }^{4}$ A. A. Balandin, S. Ghosh, W. Bao, I. Calizo, D. Teweldebrhan, F. Miao et al. Nano Lett. 8, 902 (2008).

${ }^{5}$ A. K. Geim and K. S. Novoselov, Nature. Mater. 6, 183 (2007).

${ }^{6}$ K. S. Novoselov, Z. Jiang, Y. Zhang, S. V. Morozov, H. L. Stormer, U. Zeitler, J. C. Maan, G. S. Boebinger, P. Kim, and A. K. Geim, Science 315, 1379 (2007).

${ }^{7}$ V. P. Gusynin and S. G. Sharapov, Phys. Rev. Lett. 95, 146801 (2005).

${ }^{8}$ V. P. Gusynin and S. G. Sharapov, Phys. Rev. B 71, 125124 (2005).

${ }^{9}$ M. Tahir and K. Sabeeh, J. Phys. Condens. Matter 24, 135005 (2012).

${ }^{10}$ M. Charbonneau, K. M. Van Vliet, and P. Vasilopoulos, J. Math. Phys. 23, 318 (1982); P. Vasilopoulos, Phys. Rev. B 32, 771 (1985).

${ }^{11}$ P. M. Krstajić and P. Vasilopoulos, Phys. Rev. B 83, 075427 (2011).

${ }^{12}$ Y. Zheng and T. Ando, Phys. Rev. B 65, 245420 (2002).

${ }^{13}$ M. E. Raikh and T. V. Shahbazyan, Phys. Rev. B 47, 1522 (1993).

${ }^{14}$ P. M. Krstajić and P. Vasilopoulos, Phys. Rev. B 86, 115432 (2012).

${ }^{15}$ I. Herbut, Phys. Rev. B 75, 165411 (2007).

${ }^{16} \mathrm{P}$. Phillips, Advanced Solid State Physics (Cambridge University Press, Cambridge, 2003), p. 324.

${ }^{17}$ P. M. Ostrovsky, I. V. Gornyi, and A. D. Mirlin, Phys. Rev. B 77, 195430 (2008).

${ }^{18}$ C. R. Dean, A. F. Young, I. Meric, C. Lee, L. Wang, S. Sorgenfrei, K. Watanabe, T. Taniguchi, P. Kim, K. L. Shepard, and J. Hone, Nat. Nanotechnol. 5, 722 (2010).

${ }^{19}$ J. Jung, Z. Qiao, Q. Niu, and A. H. MacDonald, Nano Lett. 12, 2936 (2012).

${ }^{20}$ L. Jiang, Y. Zheng, H. Li, and H. Shen, Nanotechnol. 21, 145703 (2010).

${ }^{21}$ G. Giovannetti, P. A. Khomyakov, G. Brocks, P. J. Kelly, and J. Brink, Phys. Rev. B 76, 073103 (2007).

${ }^{22}$ W. Gannett, W. Regan, K. Watanabe, T. Taniguchi, M. F. Crommie, and A. Zettl, Appl. Phys. Lett. 98, 242105 (2011).

${ }^{23}$ T. Seyller, A. Bostwick, K. V. Emtsev, K. Horn, L. Ley, J. L. McChesney, T. Ohta, J. D. Riley, E. Rotenberg, and F. Speck, Phys. Status Solidi B 245, 1436 (2008).

${ }^{24}$ J. Hass, W. A. de Heer, and E. H. Conrad, J. Phys.: Condens. Matter 20, 323202 (2008). 